\title{
Modal parameters identification for some sandwich bars reinforced with fiber-glass
}

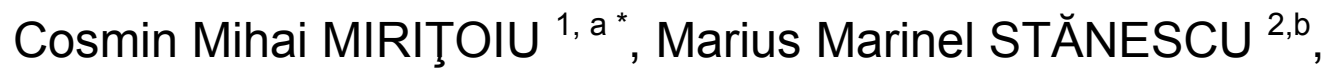 \\ Dumitru BOLCU ${ }^{3, c}$, Cristian Oliviu BURADA ${ }^{4, d}$, Vîlcu ROŞCA ${ }^{5, e}$ \\ and Sabin Vasile RIZESCU ${ }^{6, f}$ \\ ${ }^{1}$ Faculty of Mechanics, University of Craiova, 165 Calea Bucuresti, 200620, Craiova, Romania, \\ Post doctoral researcher \\ ${ }^{2}$ Department of Applied Mathematics, University of Craiova, 13 Al Cuza, 200396, Craiova, \\ Romania \\ ${ }^{3,4,5,6}$ Faculty of Mechanics, University of Craiova, 165 Calea Bucuresti, 200620, Craiova, Romania \\ a*miritoiucosmin@yahoo.com, bmamas1967@gmail.com, cdbolcu@yahoo.com, \\ dcristian.burada@yahoo.com, erosca_valcu@yahoo.com, 'sabin_rizescu@yahoo.com
}

Keywords: vibrations, sandwich bars, modal parameters, modal identification, fiber-glass.

\begin{abstract}
In this paper, there are built new composite bars with different core: with the core made of polypropylene honeycomb and polystyrene reinforced with fiber-glass. Starting from the theoretical background the modal parameters identification, there will be established an experimental method used to determine the eigenparameters of the sandwich bars. In the end, some comparisons between the eigenparameters will be made.
\end{abstract}

\section{Introduction}

The most important part of all the structures (made by men or natural ones) is the material. Usually, the improvement of the material (its chemical, mechanical and technological properties) is associated with the technological progress. New materials appear because of the necessity of a structure development or repair (to keep its initial mechanical and technological properties)[1]. Latest studies refer to materials that can decrease the structure weight and increase its strength, stiffness or vibration response. These mechanical properties provide the structure with the ability to maintain its shape under external or internal loadings. A group of materials that fulfil these conditions are the composite ones.

An important place in the group of composite materials is held by the sandwich bars and plates with constant thickness and multiple overlapped layers. Sandwich structures have many applications in the mechanical engineering, aerospace engineering, automotive engineering or civil constructions engineering, like: aircraft or satellites building, planes floor building, ships floor building, concrete forming frames or plates, vehicles body, and so on [2,3].

In this paper, there are built new composite bars with different core: with the core made of polypropylene honeycomb and polystyrene reinforced with fiber-glass. Starting from the theoretical background the modal parameters identification, there will be established an experimental method used to determine the eigenparameters of the sandwich bars. The modal identification consists in an assembly of theoretical and experimental procedures used to determine those parameters that characterize the system eigenmodes. The testing techniques can be classified in two important groups: single-point excitation and multiple-point excitation.

In this study, the single-point excitation will be used for the modal parameters identification. This method has been widely used in modal tests and it consists in applying a force in a given point and recording the vibratory structure response in all interest points, including the excitation point. Although the single-point excitation requires a minimum of equipment, it needs a laborious analysis 
to perform extensive result processing in order to interpret the dynamic behaviour of the structure under test $[4,5,6]$.

\section{Theoretical parts regarding the modal parameters identification}

The theoretical parts are presented according to the considerations made in [7], [8] and [9]. If a mechanical system is described by $N$ concentrated mass points which are jointed with elastic elements that have $\mu_{k}$ damping and $i_{k}$ stiffness, $N$ degrees of freedom, and is loaded with an external force (excitation) $\{\mathrm{P}(\mathrm{t})\}$, then the motion equations are according to Eq.1 :

$$
[G] \cdot\{y(t)\}+[\mathrm{M}] \cdot\{y(t)\}+[I] \cdot\{y(t)\}=\{P(t)\}
$$

where [G], $[\mathrm{M}]$ and $[\mathrm{I}]$ are the matrices of mass, damping and stiffness; $\{\mathrm{y}(\mathrm{t})\}$ with its first and second derivatives are the displacement, velocity and acceleration vectors; $\{\mathrm{P}(\mathrm{t})\}$ is the generalized forces vector of the external excitation. In order to determine the system response to external excitation, the Eq. 2 may be used.

$$
\sum_{j=1}^{N}\left(\frac{\left.\left\{\xi^{j}\right\} \xi^{j}\right\}^{T} \cdot\{F(\omega)\}_{j}}{-a_{j}\left(\mu_{j}-i\left(\omega+v_{j}\right)\right)}+\frac{\left.\left\{\xi^{j}\right\} \bar{\xi}^{j}\right\}^{T} \cdot\{F(\omega)\}}{-\bar{a}_{j}\left(\mu_{j}-i\left(\omega+v_{j}\right)\right)}\right)=\{Y(\omega)\}
$$

where $\{\mathrm{Y}(\omega)\}$ is the displacement Fourier transform; $\left\{\xi^{j}\right\}$ and $\left\{\xi^{j}\right\}$ is the eigenvector and its complex conjugate $j$ order; $\mu_{\mathrm{j}}$ is the damping factor $j$ order; $v_{\mathrm{j}}$ is the damped natural frequency $j$ order; $\mathrm{a}_{\mathrm{j}}$ and $\bar{a}_{j}$ are the norm constants of eigenvector; $\omega$ is the external excitation frequency.

In practice, the modal vectors can be replaced with two constants which can be obtained with Eq. 3.

$$
U_{m n}^{j}=\frac{1}{2} \cdot\left(\frac{\xi_{m}^{j} \xi_{n}^{j}}{a_{j}}+\right), V_{m n}^{j}=\frac{1}{2 \cdot i} \cdot\left(\frac{\xi_{m}^{j} \xi_{n}^{j}}{a_{j}}-\frac{\xi_{m}^{j} \xi_{n}^{j}}{a_{j}}\right)
$$

The ratio between the displacement response and the force excitation is called the system admittance and may de determined with Eq.4.

$$
\sum_{j=1}^{N}-\frac{U_{m n}^{j}+i V_{m n}^{j}}{\mu_{j}+i\left(-\omega+v_{j}\right)}-\sum_{j=1}^{N} \frac{U_{m n}^{j}+i V_{m n}^{j}}{\mu_{j}+i\left(-\omega-v_{j}\right)}=\alpha_{m n}(\omega)
$$

The concept of discrete system with concentrated mass in $N$ material points was used in the approximations adopted during the mathematical model presented in this chapter. In order to obtain an accurate approximation of the real system by the discrete one, the $N$ parameter must converge to infinity. This thing is impossible because of the experimental and processing technique and of the necessary time for data processing. So, as a consequence, the frequencies domain must be limited to a reasonable width in practical applications, which is obtained by the major resonances of the analyzed equipment and the frequency domain of the application goal. In these conditions, the sum from Eq. 4 can be reduced to several parts marked in the following stages with $N$, too. The contribution of superior and inferior modes are included in two corrections factors known as residual flexibility $\mathrm{S}_{\mathrm{ij}}^{\prime}$ (for superior modes) and inferior modal admittance $-\left(\mathrm{M}_{\mathrm{ij}}^{\prime} \cdot \omega^{2}\right)^{-1}$ (for inferior modes) (according to [7, 8, 9]). Using these notations, the Eq. 4 will have the form like in Eq. 5.

$$
\sum_{j=1}^{N}-\frac{U_{m n}^{j}+i V_{m n}^{j}}{\mu_{j}+i\left(-\omega+v_{j}\right)}-\sum_{j=1}^{N} \frac{U_{m n}^{j}+i V_{m n}^{j}}{\mu_{j}+i\left(-\omega-v_{j}\right)}+S_{m n}^{\prime}-\left(M_{m n}^{\prime} \cdot \omega^{2}\right)^{-1}=\alpha_{m n}(\omega) .
$$

So, the modal identification of a system with $N$ degrees of freedom consists in the calculus of $4 N$ modal parameters: $\mu_{j}, v_{j}, U_{m n}^{\prime}, V_{m n}^{\prime}$. These parameters are intrinsic characteristics of the system, independent of the external conditions. 


\section{Materials and experimental procedure}

There will be used composite bars with different core: with the core made of polypropylene honeycomb and polystyrene (an example is presented in Fig. 1) reinforced with fiber-glass. The connection between the fiber-glass and the core will be made by using an epoxy resin.
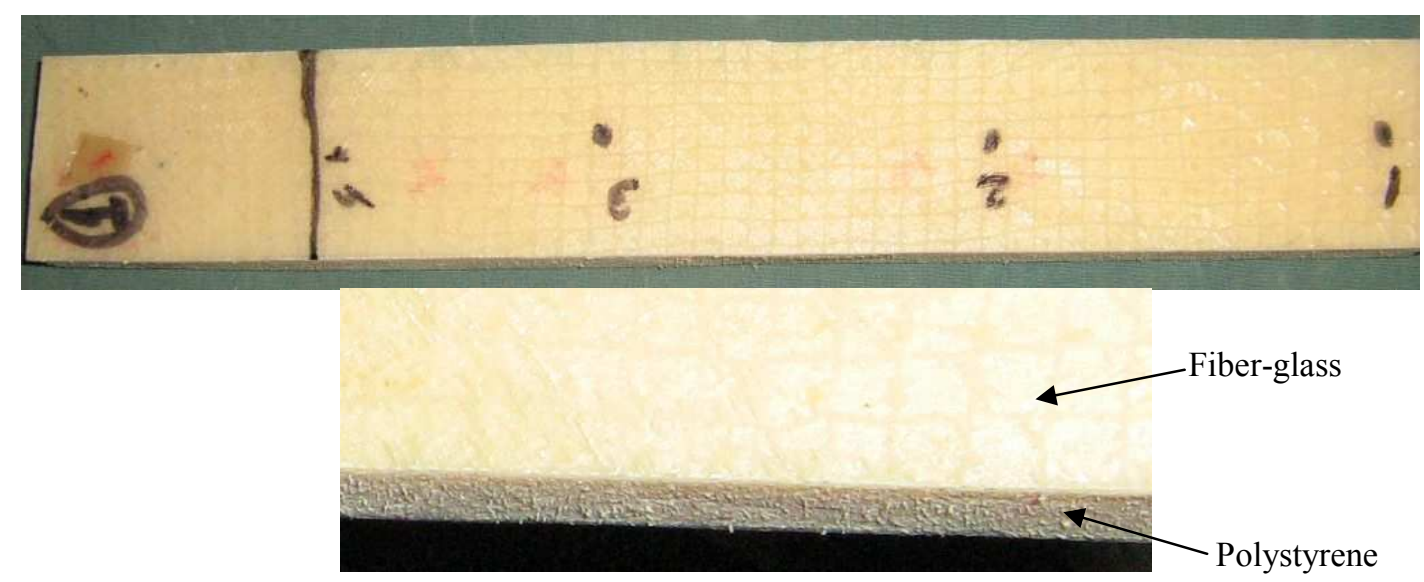

Fig. 1 General view with the sample having a polystyrene core reinforced with fiber-glass

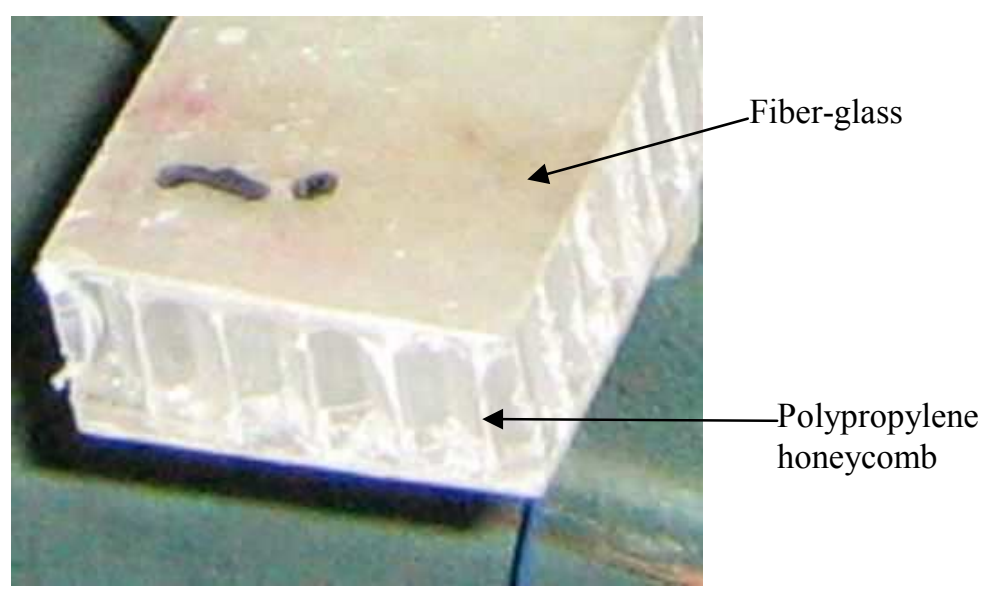

Fig. 2 General view with the sample having a polypropylene honeycomb core reinforced with fiber-glass

For the experiments, the next apparatus will be used:

- the bars will be embedded at one end and free at the other (according to the scheme from Fig. 3);

- a Bruel\&Kjaer accelerometer will be used for vibratory response recording, with $0,004 \mathrm{pc} / \mathrm{ms}^{2}$ sensitivity;

- a Bruel\&Kjaer impact hammer (having a $1,020 \mathrm{pc} / \mathrm{ms}^{2}$ sensitivity) will be used to create an excitation force, and the bars excitation will be made in the embedding area;

- for the data acquisition, a Spider 8 measuring system will be used, connected through USB port with a Fujitsu Siemens notebook;

- the accelerometers will be connected to a NEXUS signal conditioner, produced by Bruel\&Kjaer;

\section{Results and discussions}

The bar with the core made by polypropylene honeycomb will be marked as sample 1 and the bar with the core made by polystyrene will be marked as sample 2. For the sample 1, in Fig. 4 there is presented the experimental recordings for the eigenmodes determination. 


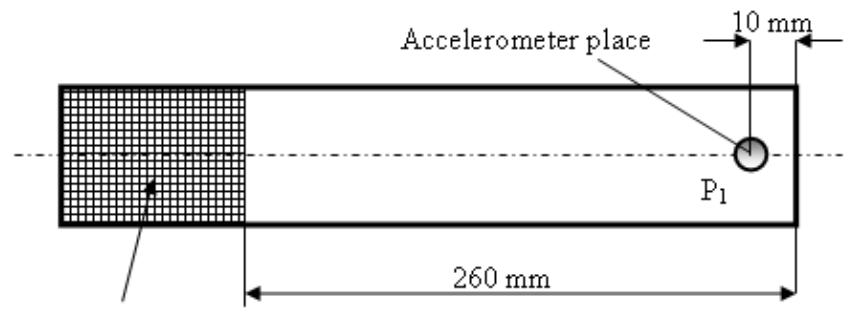

Contour embedding

Fig. 3 Experimental scheme for the sandwich bars

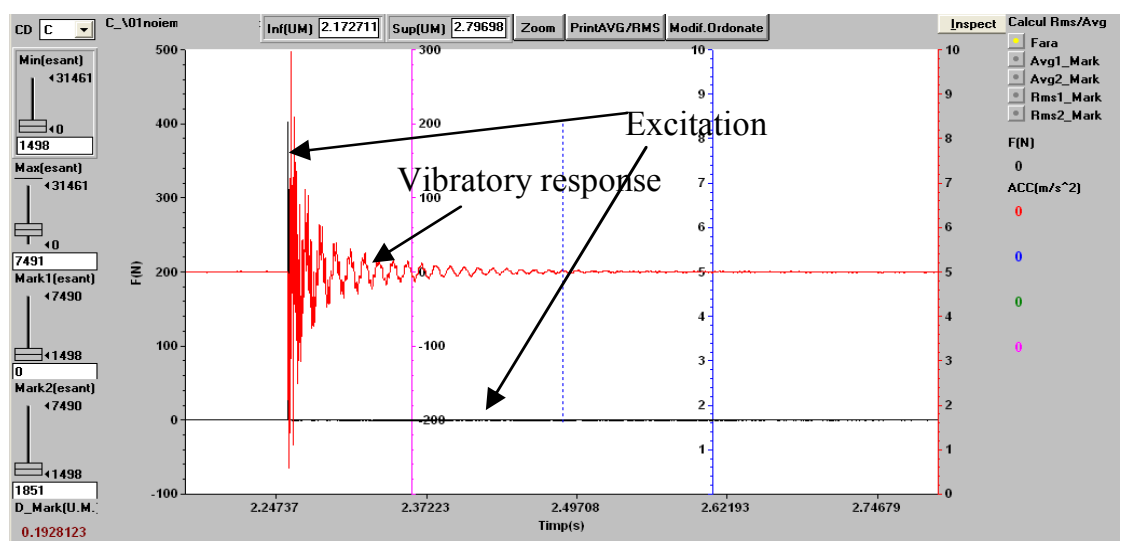

Fig. 4 Experimental recordings (sample 1)

Using the experimental recordings, the frequency response function (abbreviated in the next parts of the paper as $F R F$ ) has been determined (Fig. 5 - for Cartesian coordinates and Fig. 6 - for polar coordinates).

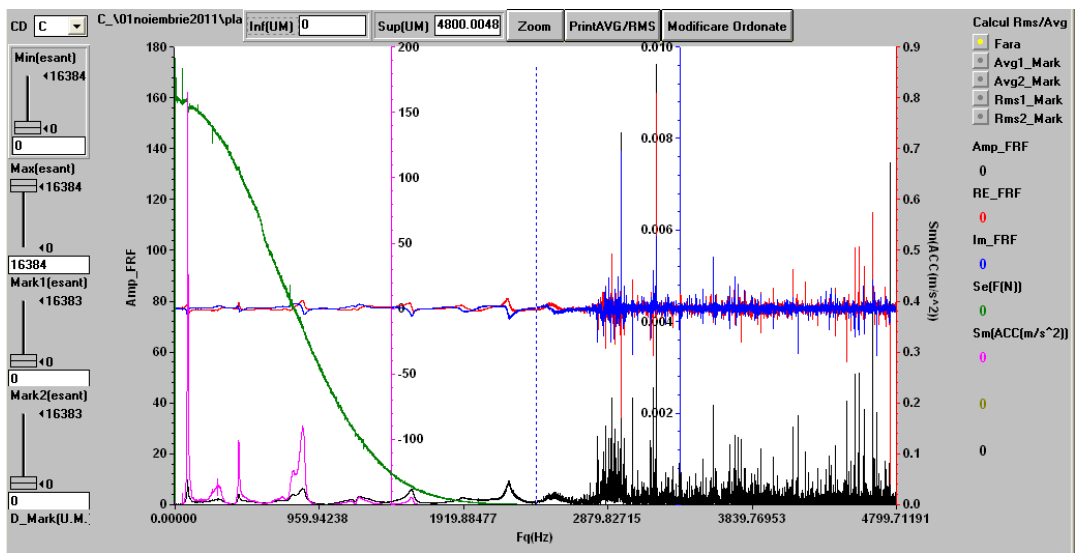

Fig. 5 FRF in the cartesian coordinates (sample 1)

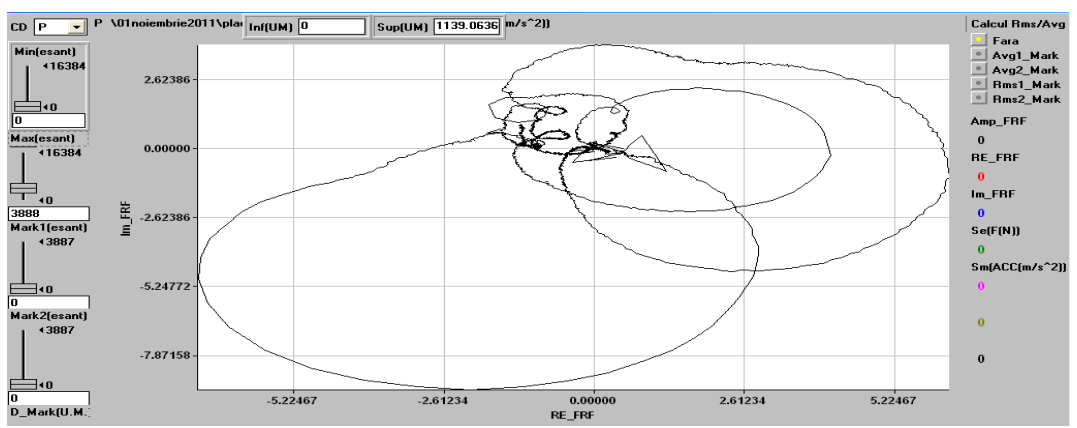

Fig. 6 FRF in the polar coordinates (sample 1) 


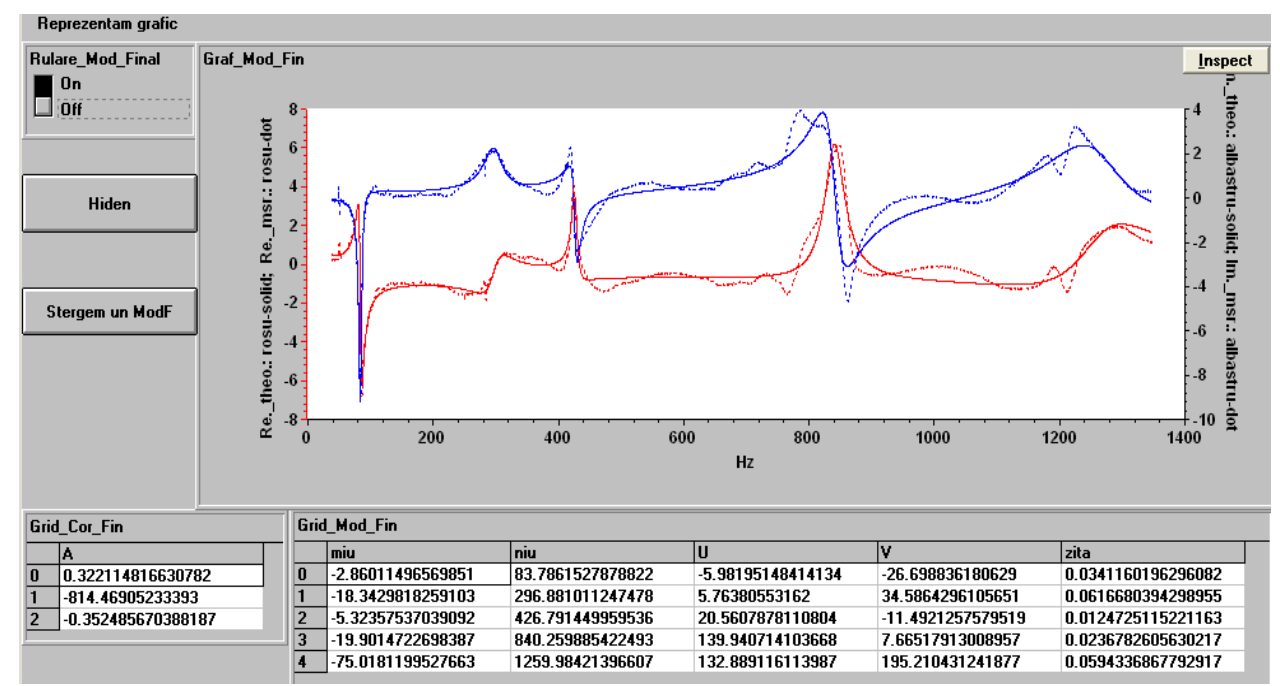

Fig. 7 Final panel with modal parameters (sample 1)

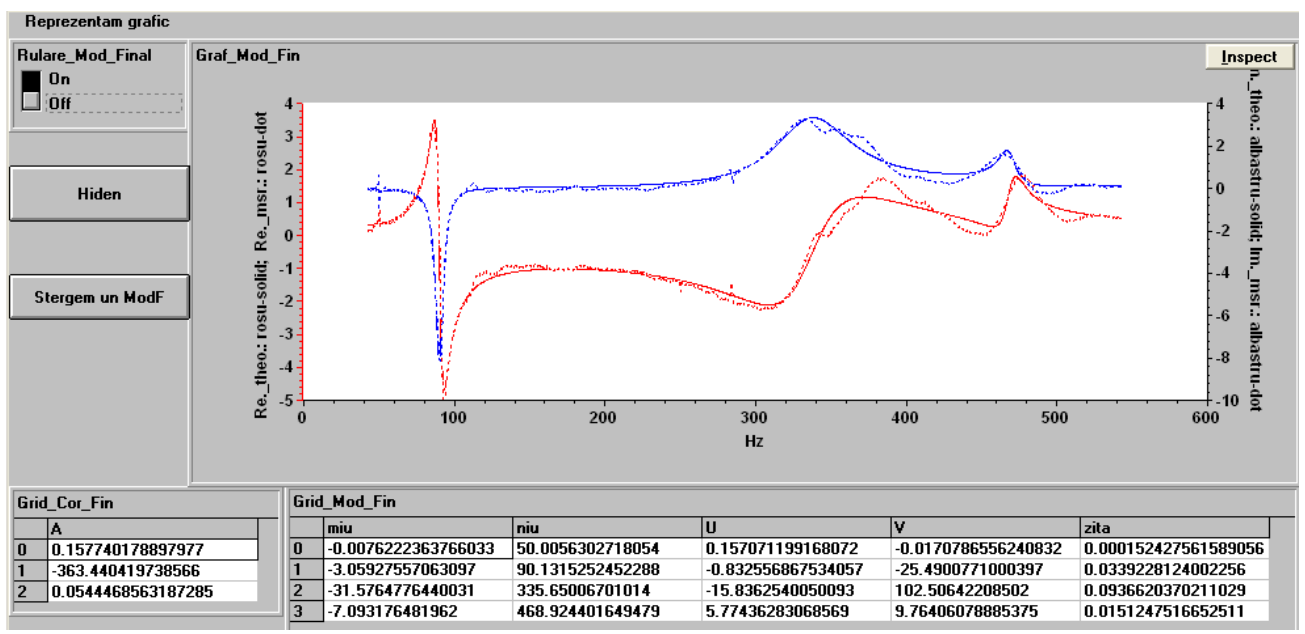

Fig. 8 Final panel with modal parameters (sample 2)

The final panel with the modal parameters is presented in Fig. 7. The same procedure was applied for the sample 2, and the final panel with the modal parameters was obtained in Fig. 8. From the Fig. 7 and Fig. 8, the next general conclusions can be extracted:

- for the sample 1, five eigenmodes were determined from the experimental setup;

- for the sample 2, four eigenmodes were determined from the experimental setup;

- for the sample 1, the eigenmodes frequencies are (measured in $\mathrm{s}^{-1}$ ): mode 1 - 83,786; mode 2 -296,881; mode 3 - 426,791; mode 4 - 840,259; mode 5-1259,984;

- for the sample 2 , the eigenmodes frequencies are (measured in $\mathrm{s}^{-1}$ ): mode $1-50,005$; mode 2 -90,131; mode 3-335,65; mode 4-468,924;

- from fig. 7 and fig. 8 , the next parameters can also be extracted: $\mu$ (miu) - the damping factor measured in $(-(\mathrm{Ns} / \mathrm{m}) / \mathrm{kg})$ and $\zeta$ (zita) - the critical damping measured in $((\mathrm{Ns} / \mathrm{m}) / \mathrm{kg})$.

\section{Conclusions}

The eigenparameters for two types of sandwich bars (the bar with the core made by polypropylene honeycomb was marked as sample 1 and the bar with the core made by polystyrene was marked as sample 2) were determined. These parameters characterize the vibratory behaviour for the considered structures.

The single-point excitation method was used for the experimental modal identification. This method consists in applying a force in a given point (in this case, in the point 4 of the composite bar, near the embedding area) and recording the vibratory structure response in all interest points, 
including the excitation point (in this case, in the point 1 , at the free end of the bar with an accelerometer having a $0,004 \mathrm{pc} / \mathrm{ms}^{-2}$ sensitivity). There have been determined five eigenmodes for sample 1 and four eigenmodes for sample 2. All the obtained results are original, because this type of the composite bar has not been studied in the engineering literature before. The above presented experiment shows many distinct eigenmodes in a large frequency domain: from $50 \mathrm{~s}^{-1}$ up to $1259 \mathrm{~s}^{-}$ 1 .

The modal identification has the advantage that is a non-destructive method and can be used for calculus of some mechanical characteristics like: stiffness, elasticity modulus, and so on. But this method also has some disadvantages like: it cannot be used to determine the maximum tensile load or the yield strength (if ductile materials are studied).

In practical engineering, these types of composites structures can be used for:

- planes floor building;

- ships floor building;

- the construction of concrete forming, and so on.

\section{Acknowledgement}

This work was supported by the strategic grant POSDRU/159/1.5/S/133255, Project ID 133255 (2014), co-financed by the European Social Fund within the Sectorial Operational Program Human Resources Development 2007-2013.

\section{References}

[1] V. Vasiliev, E. Moroyov, Mechanics and Analysis of Composite Materials, Elsevier, 0-08042702-2, Amsterdam, 2001.

[2] G. Voyiadjis, P. Katton, Mechanics of Composite Materials with MATLAB, Springer, 3-54024353-4, 2005.

[3] G. Bianchi, G. S. Aglietti, G. Richardson, Static and Fatigue Behaviour of hexagonal honeycomb cores under in-plane shear loads, Appl Comp Mater, 19 (2011), 97-115.

[4] M.M. Stanescu, D. Bolcu, I. Manea, I. Ciucă, M. Bayer, Experimental researches concerning the properties of composite materials with random distribution of reinforcement, Materiale Plastice, 46 (2009), 73-78.

[5] I. Ciucă, D. Bolcu, M.M. Stănescu, Gh. Marin, S. D. Ionescu, Study concerning some elasticity characteristics determination of composite bars, Materiale Plastice, 45 (2008), 279-280.

[6] I. Manea, D. Bolcu, C. Miriţoiu, Software for mechanical systems modal identification, Annals of the University of Craiova, Mechanical Series, 1 (2007), 191-199.

[7] D.J. Edwins, Modal Testing. Theory and Practice, Bruel\&Kjaer (1987).

[8] M.M. Stănescu, D. Bolcu, I. Manea, et. Al., Experimental Researches Concerning the Properties of Composite Materials with Random Distribution of Reinforcement, Materiale Plastice, 46:1 (2009), 73-78.

[9] I. Manea, D. Bolcu, C. Miriţoiu, Software for mechanical systems modal identification, Annals of the University of Craiova. Mechanical Series, 1 (2007), 191-199. 